\section{Posterior reversible encephalopathy syndrome and acute post-streptococcal glomerulonephritis mimicking breakthrough seizures}

\author{
Kamille Abdool, 1 \\ Kanterpersad Ramcharan, 1 \\ Neal Bhagwandass,² Navindra Persad,1 \\ Vasant Temull, 1 Karan Seegobin, 1 \\ Cassie Mike ${ }^{1}$ \\ 1Neurology Unit and 2 Nephrology Unit, \\ San Fernando Teaching Hospital, \\ University of the West Indies, San \\ Fernando, Trinidad and Tobago
}

\begin{abstract}
We report the case of a 14 -year-old boy with a past history of primary generalized seizures, who had been seizure-free for 2 years on sodium valproate and presented with generalized tonic clonic seizures suggestive of breakthrough seizures. Examination revealed hypertension, impetiginous lesions of the lower limbs, microscopic hematuria, elevated antistreptolysin 0 titre and low complement levels consistent with acute post-streptococcal glomerulonephritis. Cranial magnetic resonance imaging (MRI) demonstrated changes consistent with posterior reversible encephalopathy syndrome. Hypertension was controlled with intravenous nitroglycerin followed by oral captopril and amlodipine. Brain MRI changes returned normal within 2 weeks. The nephritis went in to remission within 2 months and after 8 months the patient has been seizure free again. Posterior reversible encephalopathy syndrome appeared to have neither short nor intermediate effect on seizure control in this patient. The relationship between posterior reversible encephalopathy syndrome and seizures is reviewed.
\end{abstract}

\section{Introduction}

Posterior reversible encephalopathy syndrome (PRES) associated with acute poststreptococcal glomerulonephritis (PSGN) or with other illnesses is rare.1,2 The risk of seizures following PRES and its association with diverse illnesses is a growing area of interest and a recently published longitudinal study demonstrated long term seizures in 3 out of 127 patients after a median follow-up of 3 years. ${ }^{3}$ We report the case of a boy with previ- ous seizures who presented clinically as possible breakthrough seizures so unmasking PRES and PSGN and focus on the relationship between seizures and PRES.

\section{Case Report}

A 14-year-old male student presented to Accident and Emergency Department with multiple seizures over a ten hour period. The semiology of seizures was typical with features of generalized tonic-clonic seizures with associated eye rolling, tongue biting, urinary incontinence and post-ictal drowsiness. All seizures were witnessed and the patient was conscious between seizure episodes. It was felt that these may have been breakthrough seizures. However, he had fever, generalized malaise, headaches, shortness of breath and productive cough for three days prior to admission. Facial and leg swelling was also reported over the same time period. No alteration in color or volume of urine output was reported and there was no visual disturbance. The patient was known to have a generalized tonicclonic seizure disorder diagnosed at age 3 years, but was seizure free for two years prior to this admission and controlled on sodium valproate $600 \mathrm{mg}$ orally twice daily. Previous brain imaging and electroencephalogram (EEG) were never done due to sociocultural reasons. There was no history of head trauma or drug abuse. He had normal milestones of development and lived in a rural forested area.

On examination, there was drowsiness (Glasgow Coma Scale 14/15) and some mild respiratory distress. Vital signs revealed an elevated blood pressure at 167/106 $\mathrm{mmHg}$, pulse $106 /$ minute, temperature $36.9^{\circ} \mathrm{C}$ with a respiratory rate of $24 \mathrm{bpm}$. Dipstick urinalysis showed 4+ blood, but no protein. The chest examination revealed crepitations bi-basally and there was obvious facial and periorbital edema. There were no focal neurological deficits or signs of meningism. Plantar reflexes were upgoing bilaterally. Pupils were $3 \mathrm{~mm}$ and reactive to light and fundoscopy was normal. On both legs there were multiple excoriated papular lesions which were attributed to scratched mosquito bites (Figure 1).

The patient was admitted to the high dependency unit and commenced on glyceryltrinitrate. Intravenous infusion (1 mg per $\mathrm{mL}$ ) given over 24 hours, which was titrated according to his blood pressure and intravenous phenytoin $100 \mathrm{mg}$ three times daily, were used in the first 24 hours to control seizures. Once stable, oral amlodipine and captopril were started, his blood pressure was controlled on these oral agents and, after day four, he remained normotensive off all antihypertensives. Sodium valproate $200 \mathrm{mg}$ orally twice
Correspondence: Kamille Abdool, Neurology Unit, San Fernando Teaching Hospital, University of the West Indies, San Fernando, Trinidad and Tobago.

Tel: +1.868.680.3791 - Fax: +1.868.657.5951.

E-mail:kamab@hotmail.com

Key words: Posterior reversible encephalopathy syndrome; post-streptococcal glomerulonephritis; breakthrough seizures; neurology.

Acknowledgements: authors would thank Dr M. Gosein for assistance with the radiological images.

Contributions: the authors contributed equally.

Conflict of interest: the authors declare no potential conflict of interest.

Received for publication: 4 April 2015. Accepted for publication: 15 April 2015.

This work is licensed under a Creative Commons Attribution NonCommercial 3.0 License (CC BYNC 3.0).

(C) Copyright et al., 2015

Licensee PAGEPress, Italy

Neurology International 2015; 7:5971

doi:10.4081/ni.2015.5971

daily was restarted on day 2 post admission. Sodium valproate level was unavailable.

Blood investigations showed normal renal and liver function tests. There was a low $\mathrm{C} 3$ level $66 \mathrm{mg} / \mathrm{dL}$ (90-180), an elevated antistreptolysin 0 titer $329 \mathrm{IU} / \mathrm{mL}(0-200)$ and normal C reactive protein. The erythrocyte sedimentation rate was $20 \mathrm{~mm} / \mathrm{hr}$ and the white cell count was elevated at 13.3/UL. Hemoglobin and platelets levels were normal. The antinuclear antibody, double stranded DNA, perinuclear anti-neutrophil cytoplasmic antibody and cytoplasm anti-neutrophil antibody were normal.

Urine microscopy revealed red blood cells: 216.3 per high power field with red cell casts. A chest X-ray was normal. Renal artery magnetic resonance angiogram, 5-hydoxyindole acetic acid, vanyllmandelic acid and metanephrine levels were all normal. Magnetic resonance imaging (MRI) changes of the brain are illustrated in Figure 2A, showing hyperintense signals bilaterally in the occipital lobes and posterior frontal/anterior parietal lobes on axial Flair image. Figure 2B shows normal $\mathrm{T}_{2}$ MRI 3 weeks later. An interictal EEG one month later was normal.

On discharge, his C3 levels had returned to normal and dipstick hematuria resolved. He was discharged on sodium valproate $200 \mathrm{mg}$ orally twice daily to outpatient care. Twelve months later the patient remained seizure free. 


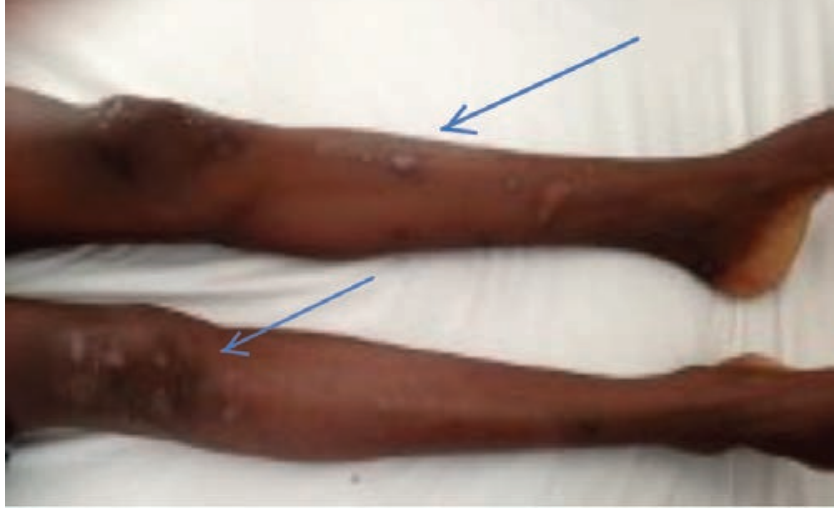

Figure 1. On both legs there were multiple excoriated papular lesions.



Figure 2. Magnetic resonance imaging of the brain: abnormal hyperintense signals seen in axial FLAIR in cortical and subcortical areas of occipital lobe and anterior parietal/posterior frontal lobes consistent with posterior reversible encephalopathy syndrome (A). Normal axial T2 image is shown 3 weeks later (B).
Written informed consent was obtained from the patient for publication of this case report and the accompanying magnetic resonance images.

\section{Discussion}

The term PRES was coined in 1996. In that report, 15 patients were evaluated and hypertensive encephalopathy was deemed the underlying cause of this syndrome. The mechanism of the syndrome is poorly understood, but it has been postulated that a brain capillary leak syndrome, fluid retention, endothelial dysfunction/injury and hypoperfusion may be the underlying pathogenetic mechanism. 4 Recently blood-brain barrier breakdown has been demonstrated in rats with decreased uterine perfusion pressure and it is hoped that this possible model of PRES will allow further elucidation of this illness. ${ }^{5}$ Our patient presented with some of the typical features of PRES, namely headaches, decreased alertness, seizures and hypertension associated with PSGN. The patient was known to have a generalized seizure disorder but had been seizure free for two years prior to presentation, demonstrating the importance of searching for new or alternative pathology in a previously well controlled seizure patient.

The relationship between seizures and PRES has aroused interest. Seizures in PRES are usually generalized and occasionally focal. Raj et al., in a study of 8 pediatric patients with PRES of diverse causes, found predominant generalized tonic and/or clonic seizures with no neurological sequel after 1 year follow up. ${ }^{6}$ PRES presenting as status epilepticus has been documented in a previously healthy child, ${ }^{7}$ two patients on chemotherapy, ${ }^{8}$ after pediatric hematopoietic stem cell transplantation in 10 patients and in a further 10 cases where focal-onset complex partial seizure was present in the majority of cases. ${ }^{9}$ PRES, in addition, can cause both convulsive and nonconvulsive status epilepticus. ${ }^{10}$

The EEG usually show diffuse theta/delta slowing and occasionally epileptogenic activity with focal sharp-wave and rarely periodic lateralizing epileptiform discharges and long term anticonvulsants are usually not required.11 Furthermore, 2 patients presenting with temporal lobe epilepsy have been found to have previous PRES,12 and occipital lobe epilepsy associated with atrophy of the occipital lobe and EEG abnormalities have been noted subsequent to PRES in post-partum eclampsia.13 Recurrent PRES in the same patient has caused chronic epilepsy. ${ }^{14}$ Also, hippocampal sclerosis has also been described in a recent case. ${ }^{15}$

Posterior reversible encephalopathy syndrome has also been recently noted to cause long term seizures albeit in low frequency. ${ }^{3}$ Long term follow up however did not show seizures as a sequel in a small cohort of patients with PRES in two other studies. 16,17

Thus the relationship between PRES and long term seizures is still unclear and the epileptogenic foci in PRES have not been mapped yet, most likely due to lack of an adequate experimental model. Our case is unusual since the patient had a history of previous generalized seizures unlike previous reported cases of PRES. It is likely that the PRES per se was responsible for the seizures leading to admission of this patient, but it is also theoretically possible that the seizure threshold at the epileptogenic zone was lowered by the preexisting seizure disorder facilitating the seizures. Our observations, though only in a singular case, show that at least in the short and medium term, typical PRES did not have a detrimental effect on seizure control.

Conceivably in cases of severe PRES with ensuing permanent brain damage, the usual seizure pattern or frequency may be altered in a patient who has preexisting seizures, given the above evidence. However, we couldn't find such reports, so this will be an area for further research. Though it appears there is full recovery in PRES in most patients with a few recorded cases of long term seizures, these reports nevertheless warn clinicians to be vigilant in diagnosis and aggressive in treatment of both hypertension and seizures in case of PRES.

\section{Conclusions}

Clinicians are therefore advised that PRES should be considered in the differential diagnosis when searching for new or underlying pathological processes during apparent breakthrough seizures; especially if predisposing factors for PRES exist and aggressive treatment should be commenced.

\section{References}

1. Adikari M, Priyangika D, Marasingha I, et al. Post-streptococcal glomerulonephritis leading to posterior reversible encephalopathy syndrome: a case report. BMC Res Notes 2014;7:644.

2. Kumar SS, Kumar M, Shobhana S, et al. Posterior reversible encephalopathy syndrome unmasking acute glomerulonephri- 
tis. J Clin Diagn Res 2014;8:177-8.

3. Datar S, Singh T, Rabinstein AA, et al. Long-term risk of seizures and epilepsy in patient swith posterior reversible encephalopathy syndrome. Epilepsia 2015; 56:564-8.

4. Hinchey J, Chaves C, Appignani B, et al. A reversible leukoencephalopathy syndrome. N Engl J Med 1996;334:494-500.

5. Porcello Marone LC, Gadonski G, de Oliveira Laguna G, et al. Blood-brain barrier breakdown in reduced uterine pressure: a possible model of posterior reversible encephalopathy syndrome. J Stroke Cerebrovasc Dis 2014;23:2075-9.

6. Raj S, Overby P, Erdfarb A, et al. Posterior reversible encephalopathy syndrome: incidence and associated factors in a pediatric critical care population. Pediatr Neurol 2013;49:335-9.

7. Mameli C, Dilillo D, Spiri D, et al. Status epilepticus as manifestation of posterior reversible encephalopathy syndrome in a healthy child. Pediatr Neurol 2013;48:41820.

8. Foreid H, Pires C, Albuquerque L, et al.
Posterior reversible encephalopathy manifested by refractory status epilepticus in two patients under chemotherapy. BMJ Case Rep 2011;2011:bcr0520114181.

9. Kozak OS, Wijdicks EF, Mannoe M, et al. Status epilepticus as initial manifestation of posterior reversible encephalopathy syndrome. Neurology 2007;68:894-7.

10. Cordelli DM, Masetti R, Bernardi B, et al. Status epilepticus as a main manifestation of posterior reversible encephalopathy syndrome after pediatric hematopoietic stem cell transplantation. Pediatr Blood Cancer 2012;58:785-90.

11. Kastrup 0, Gerwig M, Frings $M$, et al. Posterior reversible encephalopathy syndrome (PRES): electroencephalographic findings and seizure patterns. J Neurol 2012;259:1383-9.

12. Striano P, Striano S, Tortora F, et al. Clinical spectrum and critical care management of posterior reversible encephalopathy syndrome (PRES). Med Sci Monit 2005;11:CR549-53.

13. Yousaffa M, Callixte KT, Christian N. Occiptal lobe epilepsy secondary to poste- rior reversible encephalopathy syndrome (PRES) during a post-partum eclampsia in Mali (West Africa). BMC Res Notes 2013;6:321.

14. Skiba V, Etienne M, Miller JA. Development of chronic epilepsy after recurrent episodes of posterior reversible encephalopathy syndrome associated with periodic lateralized epileptiform discharges. Seizure 2012;20:93-5.

15. Kapina V, Vargas MI, Wohirab G, et al. Hippocampal sclerosis and chronic epilepsy following posterior reversible encephalopathy syndrome. Epileptic Disord 2013; 15:451-4.

16. Roth C, Ferbert A. Posterior reversible encephalopathy syndrome: long term follow up. J Neurol Neurosurg Psychiatry 2010; 81:773-7.

17. Singh RR, Ozyilmaz N, Waller S, et al. A study on clinical and radiological features and outcome in patients with posterior reversible encephalopathy syndrome (PRES). Eur J Pediatr 2014;173:1225-31. 